A N N A L E S Annales de Bretagne et des Pays de l'Ouest

Anjou. Maine. Poitou-Charente. Touraine

128-3 | 2021

Varia

\title{
Histoire de Bordeaux
}

\section{Hervé Guiet}

\section{OpenEdition}

Journals

Édition électronique

URL : https://journals.openedition.org/abpo/7094

DOI : $10.4000 / a b p o .7094$

ISSN : 2108-6443

\section{Éditeur}

Presses universitaires de Rennes

\section{Édition imprimée}

Date de publication : 29 septembre 2021

Pagination : 215-217

ISSN : 0399-0826

\section{Référence électronique}

Hervé Guiet, « Histoire de Bordeaux », Annales de Bretagne et des Pays de l'Ouest [En ligne], 128-3 | 2021, mis en ligne le 29 septembre 2021, consulté le 11 octobre 2022. URL : http://journals.openedition.org/ abpo/7094; DOI : https://doi.org/10.4000/abpo.7094 
franchissement des cours d'eau, pilotis, madriers, caissons, pour asseoir, structurer et assurer stabilité et durée dans le temps d'un ouvrage de $730 \mathrm{~m}$ de long mis en place dans le premier quart du $\mathrm{I}^{\mathrm{er}}$ siècle apr. J.-C. Sur cette longueur, l'ouvrage d'art associe le franchissement du cours d'eau à gué et section de chaussée stable avec radier de fondation et remblais d'exhaussement et de stabilisation dans une solide ossature en bois consistant en de très nombreux et pieux latéraux très resserrés (environ tous les $1,75 \mathrm{~m}$, soit au moins 630 pieux latéraux sur $550 \mathrm{~m}$ ) qui constituent un long caisson et pieux internes qui arment en la solidifiant la structure. Indépendamment de la désuétude, estimée entre le viII et le $\mathrm{x}^{\mathrm{e}}$ siècle, la qualité de cette construction est attestée par sa mention dans au moins trois chartes médiévales de donations qui enjoignent, entre autres, des obligations d'entretien. Cet ouvrage est pour le moment unique dans le monde romain occidental.

Nicolas MATHIEU

FIGEAC, Michel (dir.), Histoire de Bordeaux, Rennes, Presses universitaires de Rennes, 2019, $332 \mathrm{p}$.

Prenant place dans une collection consacrée à l'histoire des villes françaises, récemment inaugurée par Rennes (2018), le volume dédié à Bordeaux a été réalisé sous la direction de Michel Figeac, professeur d'histoire moderne à l'Université Michel de Montaigne de Bordeaux. Il réunit les contributions de onze universitaires, spécialistes des différentes périodes historiques couvertes. Ces travaux proposent une synthèse actualisée des connaissances, écrite de manière à être accessible à un large public. Le sommaire est en lui seul une invitation à voyager dans le temps, depuis la Burdigala antique jusqu'à la métropole actuelle. Le traitement sur la longue durée a imposé un plan chronologique dont le pas de temps se resserre progressivement au fil des chapitres : pluriséculaire pour l'Antiquité et le Moyen Âge, séculaire pour l'époque moderne, calé sur les temps forts économiques, politiques et militaires du XIX ${ }^{\mathrm{e}}$ au milieu du XXe siècle avant de se confondre avec l'action personnelle de deux maires à travers lesquels l'histoire récente de Bordeaux s'est incarnée pendant plusieurs décennies et qui ont imprimé leur empreinte sur la ville : Jacques Chaban-Delmas de 1947 à 1995 et Alain Juppé de 1995 à 2018. Toutefois, plus qu'un récit continu de l'histoire de la ville, l'ouvrage se présente ainsi comme une série de tableaux construits de manière autonome par chaque contributeur. On note néanmoins de leur part une nette volonté de mettre en avant la ligne de force ou le principal facteur dynamique de la période traitée : " De la ville bourgeoise à la bonne ville " pour le Moyen Âge, "Temps des réformes, temps d'épreuves vers 1530-1615 ", "Bordeaux au XVII siècle, un siècle de fer? ", " Le siècle d'or de Bordeaux (1715-1789) ", " Bordeaux de 1789 à 1815, de la cocarde aux Lys ", " Bordeaux vers une ville moderne (1815-1870) ", " Une cité-port, de l'industrie aux outre-mers (1870-1914) ", " D'une guerre à l'autre (1914-1945) ». La présentation chronologique est ainsi rythmée par des repères qui fournissent une entrée thématique au lecteur et qui, à l'échelle de l'ouvrage, recomposent les pulsations du temps long de l'histoire d'une ville en constante évolution.

Comme le signale Michel Figeac dans l'avant-propos, certains esprits chagrins pourraient marmonner qu'il s'agit là d'une Histoire de Bordeaux de plus; mais cette publication est remarquable sur plusieurs points. Tout d'abord, l'ouvrage témoigne d'un renouvellement sensible des connaissances, fruit de la fécondité des dernières décennies de recherches historiques. Ainsi, le chapitre d'Alain Bouet sur l'Antiquité et, dans une moindre mesure, celui de Bruno Dumézil sur le haut Moyen Âge sont nourris des résultats des fouilles archéologiques qui se sont multipliées depuis une 
quarantaine d'années en liaison avec les différents chantiers urbains. C'est donc une synthèse renouvelée sur les différentes évolutions de la Burdigala antique que l'on pourra lire dans ces pages. Par ailleurs, ce sont les nombreux travaux universitaires menés depuis les années 1980 - parmi lesquels les thèses des auteurs - qui ont permis de porter des regards nouveaux sur la société et l'économie de Bordeaux aux époques moderne et contemporaine. De plus, contrairement à ses devancières, dans cette Histoire de Bordeaux, les chapitres relatifs à la période la plus contemporaine n'ont pas été confiés à des géographes; Sylvie et Pierre Guillaume se sont ainsi confrontés à l'épineuse difficulté de l'écriture de l'histoire du temps présent et ce sont donc avec des perspectives historiques qu'ils ont abordé les longs mandats de Jacques Chaban-Delmas et d'Alain Juppé. Ils permettent ainsi d'appréhender les réponses complexes - parfois contradictoires - apportées à Bordeaux aux défis multiples de la modernité.

À côté de ce renouvellement des connaissances, l'ensemble des contributions témoigne des nouveaux rapports que les historiens entretiennent avec les documents iconographiques et du renouvellement des questionnements qui en découle. On ne peut ainsi qu'être sensible à la diversité des illustrations qui jalonnent l'ensemble du volume (documents d'archives, photographies diverses, cartes et plans, graphiques...) tout comme à la qualité extrême de leur reproduction. L'image n'est plus seulement illustrative, elle nourrit et construit l'analyse. C'est donc vers une histoire totale que nous conduisent les différents auteurs. Ainsi, Michel Bochaca refusant délibérément de produire un récit historique linéaire de la période médiévale - a construit sa présentation à partir d'une analyse du texte de L'Antiquité de Bourdeaux publiée par l'humaniste Elie Vinet en 1565; cette première description de Bordeaux constitue un document unique car organisé par son auteur en relation directe avec l'édition d'une vue cavalière (p. 66), sommaire soit, mais pionnière en son genre. L'histoire urbaine se lit ainsi au travers des représentations de la ville, de ses vifs pourtraicts des $\mathrm{XVI}^{\mathrm{e}}$ et $\mathrm{XVII}^{\mathrm{e}}$ siècles que reproduisent Éric Suire (p. 110 à 113) et Caroline Le Mao (p. 116). De même, le plan de Lattré gravé en 1755 et reproduit par Michel Figeac (p. 152) rend compte de l'exceptionnelle croissance de la ville de Bordeaux dans la première moitié du xvIII ${ }^{e}$ siècle. La vue cavalière retenue par Hubert Bonin (p. 218) ou le plan du guide Baedeker présentée par Philippe Chassaigne (p. 256) traduisent également la poursuite de cette dynamique tout au long du XIX ${ }^{\mathrm{e}}$ siècle. Les auteurs nous invitent donc à saisir l'évolution de la topographie de l'espace urbain dans son développement spatial. La couverture monumentale est aussi abordée. Les édifices antiques renaissent grâce aux reconstitutions informatiques, les constructions médiévales resurgissent du passé grâce aux dessins et gravures du XIX ${ }^{\mathrm{e}}$ siècle, les élégantes constructions classiques sont abondamment photographiées... Au fil de l'ensemble des contributions, le lecteur pourra à nouveau prendre conscience que la " belle endormie " qu'a été la ville de Bordeaux dans les dernières années du $\mathrm{xx}^{\mathrm{e}}$ siècle a toujours été un port - le plus actif de France au XvIII ${ }^{\mathrm{e}}$ siècle -, que cette activité maritime a fait sa force et sa richesse depuis l'Antiquité. Par les illustrations, les navires présentés par les différents contributeurs donnent à nouveau vie à la Garonne. Les vues cavalières, les huiles d'Édouard Manet ou d'Eugène Boudin ou les photographies illustrant les chapitres rédigés par Hubert Bonin offrent un regard aujourd'hui surprenant de l'intensité du trafic portuaire. L'iconographie permet de présenter également une vision dynamique des évolutions sociales à travers des représentations des hommes et des femmes laissées par chaque époque. Ce caléidoscope va des stèles funéraires d'Axula, du petit Laetus ou du sculpteur Marcus $S$. Amabilis pour l'époque antique aux émouvantes photographies contemporaines en passant par les beaux portraits de l'époque moderne et les gravures du XIX ${ }^{e}$ siècle. Bordeaux n'a jamais 
cessé d'être une capitale provinciale avant de devenir aujourd'hui une métropole régionale, de renommée internationale.

L'ouvrage, enfin, offre également une réponse historienne aux débats actifs suscités à Bordeaux par les évolutions des mémoires contemporaines. C'est avec objectivité que Michel Figeac a tenté d'aborder, dans son chapitre sur "le siècle d'or de Bordeaux ", les rapports complexes des négociants bordelais avec la traite esclavagiste (p. 158-166) ou que Philippe Chassaigne évoque les heures sombres de l'Occupation (p. 276-277). Les historiens de Bordeaux ne sont pas restés sourds aux nouveaux enjeux historiographies nés des questionnements du présent.

Ainsi, cette Histoire de Bordeaux, volumineuse (321 pages) et de grand format $(22 \times 28 \mathrm{~cm})$, est de très belle facture. Le texte est abondamment illustré à l'aide de quelques 280 figures (la majorité en couleur), d'une lisibilité remarquable et insérées dans une présentation aérée. L'ensemble est du plus bel effet. L'ouvrage se range sans conteste dans la catégorie des beaux livres, aussi plaisants à lire qu'à parcourir du regard. Le lecteur aura tout loisir de suivre la visite guidée que chaque rédacteur de chapitre lui propose, mais aussi de prendre à tout moment les chemins de traverse pour flâner librement à travers les dédales de l'histoire de Bordeaux, comme il le ferait en se promenant au hasard des rues du centre historique. Au-delà de l'histoire de Bordeaux, ce livre sonde la mémoire de la ville, on serait presque tenté de dire son âme.

Hervé Guiet

VIGIER, Fabrice (dir.), Poitiers, capitale de province. Essai d'histoire administrative, du $I^{\text {er }}$ siècle à 2015, Poitiers, Atlantique, 2021, 221 p.

Pour mettre en perspective l'histoire de Poitiers comme capitale provinciale, peu après que la cité eut perdu son statut de chef-lieu de la région Poitou-Charentes, le $1^{\mathrm{er}}$ janvier 2016, la Société des Antiquaires de l'Ouest organisa en septembre 2019 à la mairie de Poitiers une table ronde intitulée "Poitiers, capitale de province de la période antique à 2015 ". Le succès de cette manifestation incita ses organisateurs à en tirer un livre qui est divisé en cinq chapitres, correspondant chacun à cinq périodes : "Poitiers antique : capitale ou ville moyenne? " (Jean Hiernard), "Poitiers, une capitale régionale au Moyen Âge " (Robert Favreau), "Poitiers aux $\mathrm{XVI}^{\mathrm{e}}, \mathrm{XVII}^{\mathrm{e}}$ et XVIII ${ }^{\mathrm{e}}$ siècles : une cité opportuniste, combative et toujours ambitieuse " (Fabrice Vigier), "Poitiers aux XIX et XX ${ }^{\mathrm{e}}$ siècles : permanences et adaptations d'une capitale régionale " (Didier Veillon), et " Poitiers, héritière sans testament " (Pascal Chauchefoin) qui présente une analyse de la situation actuelle de la ville. La conclusion est une synthèse des cinq communications qui s'interroge sur les raisons pour lesquelles Poitiers fut capitale provinciale pendant plusieurs siècles (Fabrice Vigier et Stéphane Mottet). L'ouvrage est enrichi de 25 cartes très lisibles qui permettent de visualiser l'évolution des limites géographiques des différentes circonscriptions placées sous la tutelle de Poitiers, d'une utile chronologie (p. 191-197) et d'une bibliographie classée par ouvrages généraux et par périodes (p. 198-216).

Poitiers fut une capitale de province du IX ${ }^{\mathrm{e}}$ siècle à 2015 , sauf pendant trois brèves périodes qui apparaissent comme des " ruptures " dans son histoire politique et institutionnelle. En 1204, elle fut rattachée, avec le comté de Poitou, à la couronne de France; en 1436, après avoir été pendant dix-huit ans la deuxième capitale du " royaume de Bourges ", elle vit le Parlement, la chancellerie et la cour des aides rejoindre Paris; en 1790-1799, elle perdit toutes les institutions d'Ancien Régime qu'elle abritait depuis des siècles : généralité, sénéchaussée, présidial, gouvernement, université. 